\title{
Microbiology Specimen Timepoint
}

National Cancer Institute

\section{Source}

National Cancer Institute. Microbiology Specimen Timepoint. NCI Thesaurus. Code

C87922.

A point in time referring to microbiology specimen data. 